\title{
Brolucizumab in the Treatment of Neovascular Age-related Macular Degeneration
}

\author{
An Expert Interview with Pravin U Dugel
}

Retinal Consultants of Arizona, Phoenix, AZ; University of South Carolina Roski Eye Institute, Keck School of Medicine, Los Angeles, CA, US

DOI: https://doi.org/10.17925/USOR.2018.11.2.75

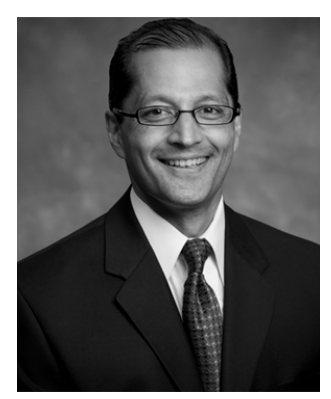

\section{Pravin U Dugel}

Pravin U Dugel graduated Summa Cum Laude from Columbia University in the City of New York, US, before attending University of California, LOS Angeles (UCLA) School of Medicine, LOS Angeles, CA, US. He completed his residency in ophthalmology at the Roski Eye Institute, Keck School of Medicine, CA, US. He completed his medical retina fellowship at the Bascom Palmer Eye Institute and his surgical retina fellowship at the Roski Eye Institute, where he was elected to serve on the faculty as the Resident Director. Dr Dugel joined Retinal Consultants of Arizona in July 1994 and is currently managing partner at Retinal Consultants of Arizona. He also continues to work with the Retinal Research Institute and is clinical professor at the Roski Eye Institute, Keck School of Medicine, University of Southern California, as well as a founding member of the Spectra Eye Institute, Sun City, and physician executive director at the Banner Eye Institute, Banner University Medical Center, University of Arizona, AZ, US.

\section{Keywords}

Neovascular age-related macular degeneration, brolucizumab, HAWK, HARRIER, anti-VEGF, vascular endothelial growth factor

\section{Disclosure: Pravin U Dugel is a}

consultant for Alcon and Novartis.

Acknowledgement: Medical writing assistance was provided by Katrina Mountfort of Touch Medical Media.

Review Process: This is an expert interview and as such has not undergone the journal's standard peer review process.

Authorship: The named author meets the Internationa Committee of Medical Journal Editors (ICMJE) criteria for authorship of this manuscript, takes responsibility for the integrity of the work as a whole, and has given final approval for the version to be published.

open Access: This article is published under the Creative Commons Attribution Non-commercial License, which permits any non-commercial use, distribution, adaptation, and reproduction provided the original author and source are given appropriate credit. (C) The Author 2018.

Received: July 26, 2018

Published Online: October 15, 2018

Citation: US Ophthalmic Review, 2018;11(2):75-6

Corresponding Author: Pravin U Dugel, Retinal Consultants of Arizona, 15401 North 29th Avenue Phoenix, AZ 85053, US. E: pdugel@gmail.com

Support: No funding was received in

the publication of this article.
$\mathrm{N}$ eovascular age-related macular degeneration (nAMD) is the leading cause of severe vision loss and irreversible blindness in people aged over 65 years in North America, Europe, Australia, and Asia. ${ }^{1}$ Anti-vascular endothelial growth factor (anti-VEGF) therapies have led to great improvements in outcomes for patients with nAMD. ${ }^{2}$ However, currently available anti-VEGF treatments require frequent clinic visits to monitor patient response to treatment and, in some cases, monthly or bimonthly intravitreal injections; a substantial treatment burden for patients and healthcare providers. ${ }^{2}$ Brolucizumab (RTH258) is a humanized single-chain antibody fragment, and is a small molecule $(26 \mathrm{kDa})$ that has shown potent inhibition of, and high affinity to, all VEGF-A isoforms. ${ }^{3,4}$ Its small size allows enhanced tissue penetration and rapid clearance from the circulation. ${ }^{4}$ The HAWK (NCT02307682)5 and HARRIER (NCT02434328) ${ }^{6}$ trials are phase III, 96-week, double-masked, randomized, prospective, multicentre studies investigating the efficacy and safety of brolucizumab compared with aflibercept in patients with nAMD. Primary data from these studies were presented at the American Academy of Ophthalmology (AAO) 2017 Annual Meeting on November 10, 2017, in New Orleans, US. ${ }^{7}$ Data from a pre-specified analysis of the HAWK and HARRIER studies were presented at the Association for Research in Vision and Ophthalmology (ARVO) 2018 Annual Meeting, Honolulu, Hawaii. ${ }^{8}$ In an expert interview, Dr Dugel discusses these data.

\section{Q. What are the major unmet needs in the treatment of nAMD?}

There are three major areas of unmet need. The first is improved efficacy in the short term, then increased durability of treatment, and finally maintenance and even improvement of efficacy in the long term. A drug or device that could address one of these three areas would be valuable; anything that could address two or three would be extremely valuable.

\section{Q. How does brolucizumab differ from other anti-VEGF treatment options in nAMD?}

Firstly, the structure of the molecule is unique. It is the first single-chain monoclonal antibody fragment; this contains only the active end of the molecule but not the constant repeats. From a clinical aspect, this allows a higher molar concentration because it is such a small molecule. As a result, the treatment interval can be extended for more patients. I think of it as the next-generation anti-VEGF therapy. 


\section{Q. What were the major findings of the HAWK and HARRIER studies comparing brolucizumab with aflibercept in patients with nAMD?}

Brolucizumab met its primary endpoint of non-inferiority at week 48. Most of the patients were dosed every 12 weeks compared with every 8 weeks on-label treatment for aflibercept. Most importantly, in the comparison at week 16 where the dosing regime was identical in the two treatment arms, brolucizumab was superior to aflibercept in every anatomical (i.e., structural optical coherencetomography) parameter thatwas measured. For instance, in resolving intraretinal and/or sub-retinal fluid, brolucizumab was superior both at week 16 (the head-to-head comparison) and week 48 (the primary endpoint). When disease activity was assessed by an investigator, brolucizumab was also superior.

\section{Q. Could you tell us about the recent analysis of the HAWK and HARRIER data investigating the reliability of response to an initial 12-week treatment interval in predicting long-term response to this dosing regimen?}

Not all patients respond the same way to treatments for nAMD; there is a wide variability in the disease. One unique aspect of the HAWK and HARRIER studies was that they were the first phase III studies to address this variability and the need for individualized treatments. Disease activity assessments were done by the masked investigator at various pre-specified time points to be certain that the appropriate patients were being extended to 12 weeks. If any of these disease activities were present, the patient was adjusted to an 8-week dosing interval for the remainder of the studies. There were numerous opportunities for the masked investigator to adjust dosing intervals to 8 weeks. Results showed that, after the first adjustment following the first cycle of 12 -week dosing, more than $80 \%$ of patients continued on this regimen up to week 48 . This suggests that, once the patients have been selected as appropriate for 12-week dosing, they tend to remain on this regimen.?

\section{Q. What are the next steps in the clinical development of brolucizumab for nAMD?}

Novartis intends to file for approval in the US at the end of this calendar year. Another study (NCT03386474) ${ }^{9}$ has recently completed recruitment.

\section{Q. What will this mean in terms of your clinical practice?}

As a clinician, I like to have choices, and ultimately we are dictated by our preferences, our patients' preferences and, most importantly, the disease itself. We are looking for a drug that is most efficient in suppressing a validated target, VEGF-A. The week 16 data of HAWK and HARRIER show that brolucizumab is more efficient than aflibercept in suppressing VEGF-A. When the drug becomes available, I believe it will be very valuable for the treatment of $\mathrm{nAMD}$. $\square$
1. Jonas JB, Bourne RR, White RA, et al. Visual impairment and blindness due to macular diseases globally: a systematic review and meta-analysis. Am J Ophthalmol. 2014;158:808-15.

2. Ishikawa M, Jin D, Sawada Y, et al. Future therapies of wet age-related macular degeneration. J Ophthalmo 2015;2015:138070.

3. Tietz J, Spohn G, Schmid G, et al. Affinity and potency of RTH258 (ESBA1008), a novel inhibitor of vascular endothelial growth factor a for the treatment of retinal disorders. Investigative Ophthalmology \& Visual Science. 2015;56:1501.

4. Gaudreault J, Gunde T, Floyd HS, et al. Preclinical pharmacology and safety of ESBA1008, a single-chain antibody fragment, investigated as potential treatment for age related macular degeneration. Investigative Ophthalmology \& Visual Science. 2012;53:3025.

5. Clinicaltrials.gov. Efficacy and Safety of RTH258 Versus Aflibercept. ClinicalTrials.gov Identifier: NCT02307682. Available at: https:// clinicaltrials.gov/ct2/show/NCT02307682 (accessed September 3, 2018).

6. Clinicaltrials. gov. Efficacy and Safety of RTH258 Versus Aflibercept - Study 2. ClinicalTrials.gov Identifier: NCT02434328. Available at: https://clinicaltrials.gov/ct2/show/NCT02434328 (accessed September 3, 2018).

7. Dugel P, et al. HAWK \& HARRIER: 48-week results of 2 multicentered, randomized, double-masked trials of brolucizumab versus aflibercept for neovascular AMD. Presented at the
American Academy of Ophthalmology 2017 Annual Meeting November 10, 2017, New Orleans, US

8. Dugel P, et al. A Comparison of the anatomical efficacy of brolucizumab and aflibercept in neovascular age-related macular degeneration (nAMD): an analysis over 16 weeks of matched treatment in the HAWK and HARRIER studies. Presented at the treatment in the HAWK and HARRIER studles. Presented at the Assoiation for Reser. 2018 Annua Meeting, Apri $29-M a y 3,2018$, Honolulu, HI, US. 9. Clinicaltrials. gov. Study of Safety and Efficacy of Brolucizumab $6 \mathrm{mg}$ Drug Product Intended for Commercialization in Patients With nAMD. ClinicalTrials.gov Identifier: NCT03386474. Available at: https://clinicaltrials.gov/ct2/show/NCT03386474 (accessed September 3, 2018) 